Western University

Scholarship@Western

Medical Biophysics Publications

Medical Biophysics Department

4-11-2015

\title{
Survival prediction in high-grade gliomas using CT perfusion imaging.
}

Timothy Pok Chi Yeung

Yong Wang

Wenqing $\mathrm{He}$

Benedetta Urbini

Roberta Gafà

See next page for additional authors

Follow this and additional works at: https://ir.lib.uwo.ca/biophysicspub

Part of the Medical Biophysics Commons

Citation of this paper:

Yeung, Timothy Pok Chi; Wang, Yong; He, Wenqing; Urbini, Benedetta; Gafà, Roberta; Ulazzi, Linda; Yartsev, Slav; Bauman, Glenn; Lee, Ting-Yim; and Fainardi, Enrico, "Survival prediction in high-grade gliomas using CT perfusion imaging." (2015). Medical Biophysics Publications. 67.

https://ir.lib.uwo.ca/biophysicspub/67 


\section{Authors}

Timothy Pok Chi Yeung, Yong Wang, Wenqing He, Benedetta Urbini, Roberta Gafà, Linda Ulazzi, Slav Yartsev, Glenn Bauman, Ting-Yim Lee, and Enrico Fainardi 


\title{
Survival prediction in high-grade gliomas using CT perfusion imaging
}

\author{
Timothy Pok Chi Yeung ${ }^{1,2,6} \cdot$ Yong Wang ${ }^{2}$ - Wenqing $\mathrm{He}^{4} \cdot$ Benedetta Urbini $^{5}$. \\ Roberta Gafà ${ }^{10}$ - Linda Ulazzi ${ }^{10}$ - Slav Yartsev ${ }^{1,3,6}$. Glenn Bauman ${ }^{1,3,6}$. \\ Ting-Yim Lee ${ }^{1,2,3,7,8} \cdot$ Enrico Fainardi ${ }^{9}$ The Project of Emilia-Romagna Region on \\ Neuro-Oncology (PERNO) Study Group
}

Received: 1 September 2014/ Accepted: 2 April 2015

(C) Springer Science+Business Media New York 2015

\begin{abstract}
Patients with high-grade gliomas usually have heterogeneous response to surgery and chemoirradiation. The objectives of this study were (1) to evaluate serial changes in tumor volume and perfusion imaging parameters and (2) to determine the value of these data in predicting overall survival (OS). Twenty-nine patients with World Health Organization grades III and IV gliomas underwent magnetic resonance (MR) and computed tomography $(\mathrm{CT})$ perfusion examinations before surgery, and 1 , $3,6,9$, and 12 months after radiotherapy. Serial measurements of tumor volumes and perfusion parameters were evaluated by receiver operating characteristic analysis, Cox proportional hazards regression, and Kaplan-Meier survival analysis to determine their values in predicting OS. Higher trends in blood flow (BF), blood volume (BV), and
\end{abstract}

A complete list of the members of the PERNO study group appears in the "Appendix".

Electronic supplementary material The online version of this article (doi:10.1007/s11060-015-1766-5) contains supplementary material, which is available to authorized users.

Ting-Yim Lee

tlee@ robarts.ca

1 Department of Medical Biophysics, Western University, London, ON, Canada

2 Robarts Research Institute, P.O. Box 5015, 100 Perth Drive, London, ON, Canada

3 Department of Oncology, Western University, London, ON, Canada

4 Department of Statistical and Actuarial Sciences, Western University, London, ON, Canada

5 Oncology Unit, Specialized Medical Department, Azienda Ospedaliero-Universitaria, Arcispedale S. Anna, Ferrara, Italy permeability-surface area product in the contrast-enhancing lesions (CEL) and the non-enhancing lesions (NEL) were found in patients with OS $<18$ months compared to those with $\mathrm{OS} \geq 18$ months, and these values were significant at selected time points $(P<0.05)$. Only CT perfusion parameters yielded sensitivities and specificities of $\geq 70 \%$ in predicting 18 and 24 months OS. Pre-surgery BF in the NEL and BV in the CEL and NEL 3 months after radiotherapy had sensitivities and specificities $>80 \%$ in predicting 24 months OS in patients with grade IV gliomas. Our study indicated that CT perfusion parameters were predictive of survival and could be useful in assessing early response and in selecting adjuvant treatment to prolong survival if verified in a larger cohort of patients.

Keywords High-grade gliomas - Glioblastoma multiforme $\cdot$ Computed tomography $\cdot$ CT perfusion . Overall survival

6 London Regional Cancer Program, London Health Sciences Centre, London, ON, Canada

7 Department of Medical Imaging, Western University, London, ON, Canada

8 Lawson Health Research Institute, London, ON, Canada

9 Neuroradiology Unit, Department of Neurosciences and Rehabilitation, Azienda Ospedaliero-Universitaria, Arcispedale S. Anna, Ferrara, Italy

10 Section of Pathology and Biomolecular Diagnostics, Department of Morphology, Surgery and Experimental Medicine, University of Ferrara, Ferrara, Italy 


\section{Introduction}

High-grade gliomas account for over $70 \%$ of all malignant brain tumors, and survival remains dismal even after maximal safe resection, radiotherapy, and temozolomide chemotherapy. The median survival is typically $12-15$ months and 2-5 years for patients with World Health Organization (WHO) grade IV and grade III gliomas, respectively [1]. However, there is considerable heterogeneity in patients' treatment response. At present, widely accepted prognostic factors of survival in high-grade gliomas are WHO grade, extent of surgical resection, age, and performance status [2]. Molecular biomarkers, such as isocitrate dehydrogenase 1 (IDH1) mutation status, O6-methylguanine-DNA-methyltransferase (MGMT) methylation status and 1p19q loss of heterozygosity are also recognized as prognostic biomarkers in these patients [3].

Magnetic resonance (MR) is the standard imaging method for assessing high-grade gliomas [4]. For radiographic assessment, the typical MR protocol includes a T2weighted or fluid-attenuated inversion recovery (FLAIR) MR and a gadolinium-enhanced T1-weighted MR [5]. The gadolinium-enhanced T1-weighted MR images depict the contrast-enhancing lesion (CEL) with disrupted bloodbrain barrier. The T2-weighted or FLAIR MR images detect regions of $\mathrm{T} 2$ hyperintensity that are suspicious of tumor infiltration and vasogenic edema. This T2 hyperintense region is the non-enhancing lesion (NEL).

In addition to anatomical imaging, functional imaging, such as perfusion imaging, provides quantitative information regarding tumor biology that may be related to patient prognosis [6]. Measurements of tumor blood flow (BF), blood volume (BV), and permeability obtained by perfusion imaging have been shown to correlate with WHO grade [7, 8], histopathologic marker of tumor angiogenesis (e.g. microvessel density) [9,10], and outcome [11-13]. Most perfusion imaging studies that evaluated the relationships between tumor perfusion parameters and overall survival (OS) have focused on MR perfusion imaging at a single time point [14] or two time points [15]. CT perfusion has not been studied extensively despite the ubiquity of CT scanners [16]. However, CT perfusion can help distinguish between high and low grade gliomas [7, 17-19] and between tumor recurrence and radiation necrosis $[20,21]$. Therefore, CT perfusion should be considered as a potentially valuable perfusion imaging modality in neuro-oncology. To our knowledge this study is the first to investigate serial changes in tumor BF, BV, and permeability-surface area (PS) in the CEL and NEL of patients with high-grade gliomas for up to a year after radiotherapy. In this analysis, we used these data to evaluate the prognostic value of CT perfusion imaging in predicting OS of these patients.

\section{Materials and methods}

\section{Patient population}

This study was conducted in compliance with the institutional research ethics committee, and informed consent was obtained from patients. Patients with suspected WHO grade III and IV gliomas were prospectively recruited prior to surgery. Exclusion criteria were (1) prior diagnosis or therapy of a brain lesion, (2) clinically unstable, (3) contraindications to contrast materials, and (4) contraindications to MR imaging such as metallic implants, claustrophobia, and obesity. Our study included 29 patients with high-grade gliomas. The median age at diagnosis was 61 (range 31-81) years. Patients underwent serial MR and CT perfusion examinations prior to surgery and one, three, six, nine, and 12 months after radiotherapy. After surgery, genomic DNA was extracted from formalin-fixed paraffinembedded tissue samples using a DNA extraction kit (Qiagen, Germany) after micro-dissection. MGMT promoter methylation status was determined for WHO grade IV glioma patients by methylation specific polymerase chain reaction after prior DNA bisulfite modification [22]. For WHO grade III glioma patients, IDH1 mutation was assessed by exon 4 direct DNA sequencing [23]. Primer extension sequencing was performed with the use of the Big Dye Terminator v1.1 Cycle Sequencing Kit (Applied Biosystems, Foster City, CA, USA). Sequences were determined using the ABI PRISM 310 Genetic Analyzer (Applied Biosystems) and the Sequencing analysis 5.2 software (Applied Biosystems).

All patients underwent surgery followed by radiotherapy (60 Gy in 30 fractions, $N=28$; 45 Gy in 15 fractions with stereotactic boost of $24 \mathrm{~Gy}$ in 3 fractions, $N=1$ ). Twentysix patients received concurrent and adjuvant temozolomide chemotherapy. Six patients underwent second surgical resection after radiotherapy, and corticosteroids were administered to patients before the second surgery. WHO grade IV patients were also given Fotemustine at the time of progression. The median follow-up was 18.2 (range, 4.7-60.4) months.

\section{$M R$ and $C T$ perfusion examinations}

MR images were acquired with a $1.5 \mathrm{~T}$ Signa HDXT (GE Healthcare, Milwaukee, WI) or a $1.5 \mathrm{~T}$ Achieva scanner (Philips Medical Systems, Best, The Netherlands). The MR protocol included the following sequences: axial T1weighted spin-echo, axial T2-weighted spin-echo or axial FLAIR, coronal FLAIR, and post-gadolinium axial T1weighted spin echo.

All CT perfusion studies were performed using a multidetector-row CT scanner (Lightspeed VCT, GE) that 
covered eight 5-mm sections of tissue. Ten patients were imaged with a one-phase CT perfusion protocol while 19 patients were imaged with a two-phase CT perfusion protocol. For the one-phase protocol, a bolus of non-ionic contrast (Iomeron, Bracco Imaging, Konstanz, Germany; $350 \mathrm{mg} \mathrm{I} / \mathrm{ml}, 40 \mathrm{ml}$ ) was injected at a rate of $4 \mathrm{~mL} / \mathrm{s}$. A cine scan of $50 \mathrm{~s}$ duration was initiated at $5 \mathrm{~s}$ after the injection $(100 \mathrm{~mA}, 80 \mathrm{kV}, 1$ rotation/s), and the images were reconstructed at $0.5 \mathrm{~s}$ intervals. For the two-phase protocol, images were reconstructed at $0.5 \mathrm{~s}$ intervals in the initial $44 \mathrm{~s}$ cine scan, followed by eight additional axial images at $15 \mathrm{~s}$ intervals for another $105 \mathrm{~s}$. The total scan duration was $150 \mathrm{~s}$.

\section{Image analysis}

Maps of BF, BV, and PS were computed using CT Perfusion 4D (GE Healthcare) [24, 25]. Averaged CT images were produced by averaging the cine $\mathrm{CT}$ images of the same sections. MR images were rigidly registered to the averaged CT images using 3D Slicer [26]. A radiologist with 8 years of experience delineated the entire contrastenhancing lesion (CEL) and the entire non-enhancing lesion (NEL). The NEL was the peritumoral T2 hyperintense region outside the CEL, central necrosis, and surgical cavity. Thus, the NEL included peritumoral edema and post-radiation T2 changes around the CEL. Mean volume, $\mathrm{BF}, \mathrm{BV}$, and PS in both the CEL and NEL were used for analysis.

\section{Statistical analysis}

Statistical analysis was performed using SPSS (IBM ${ }^{\circledR}$ SPSS $^{\circledR}$, version 21.0, Chicago, IL) and R (http://www.rproject.org/) to evaluate whether the imaging parameters were candidate biomarkers of OS. Patients were stratified by their OS using 12, 18, and 24 months OS as endpoints. Patients were designated as "long-term survivors" (OS $\geq 18$ months) $\quad$ or "short-term survivors" (OS $<$ 18 months) based on the 18 months OS endpoint. The imaging parameters in patients with short-term versus long-term OS were examined longitudinally. The Friedman test followed by the Wilcoxon signed-rank test were used for longitudinal comparisons within-group, and the MannWhitney $U$ test examined the differences between groups. Only imaging data from pre-surgery, one, three, and 6 months post-radiotherapy were included for analysis due to patient dropouts resulting from deaths or complete regression of tumors at nine and 12 months postradiotherapy.

For the entire cohort of 29 patients, receiver operating characteristic analyses were performed to consider all possible cut points to differentiate patients with long-term and short-term OS. The optimal cut point for each imaging parameter was selected for use in Cox proportional hazards regression and Kaplan-Meier survival analyses. Cox proportional hazards regression analyses were performed to determine the relationships between OS and the imaging parameters. A separate Cox proportional hazards regression model was computed for each imaging parameter at each time point while adjusting for age, Karnofsky performance status, extent of surgical resection, and WHO grade. The hazard ratios (HR) and their $95 \%$ confidence intervals (CI) were computed. IDH1 mutation status was not adjusted in the Cox proportional hazards regression due to the small number of WHO grade III patients $(N=5)$. Kaplan-Meier survival analysis and log-rank test compared the OS of patients with high versus low values of imaging parameters.

For patients with grade IV gliomas $(N=24)$, Cox proportional hazards regression and Kaplan-Meier survival analysis were repeated. MGMT promoter status, age, Karnofsky performance status, and the extent of resection were also included in the Cox proportional hazards regression. A $P \leq 0.05$ was considered statistically significant. Lastly, joint modeling of OS and the longitudinal covariates was performed to evaluate whether changes in these imaging parameters correlate with OS [27].

\section{Results}

\section{Patient characteristics and outcomes}

A total of $150 \mathrm{CT}$ perfusion and $150 \mathrm{MR}$ examinations were acquired, and $109 \mathrm{CT}$ perfusion and $109 \mathrm{MR}$ scans from the first 4 times points were analyzed. Twenty-two patients had imaging studies available from the first four time points while seven patients had imaging studies available from the first three time points. No radiationinduced side effects (e.g. skin erythema) from the serial CT perfusion scans was noted. The median OS was 18 months (range, 5-60 months) with three patients alive at the end of the study. Table 1 summarizes patient characteristics and the corresponding OS data. In univariate analyses, gender, extent of surgical resection, Karnofsky performance status, re-operation, and second-line Fotemustine were not significant predictors of OS. Older patients ( $\geq 50$ years) had worse OS than younger patients, but this was marginally significant $(P=0.06)$. WHO grade IV was associated with worse OS when compared to grade III $(P=0.04)$, while patients with unmethylated MGMT promoter status was associated with worse OS compared to patients with methylated MGMT promoter $(P<0.001)$. IDH1 mutation could be associated with OS $(P=0.05)$, but the sample size was too small to be conclusive (wild-type $N=1$ ). 
Table 1 Patient characteristics, percentages of patients living beyond 12, 18, and 24 months, and median OS

\begin{tabular}{|c|c|c|c|c|c|c|}
\hline Demographics & $n$ & 12 Months OS (\%) & 18 Months OS (\%) & 24 Months OS (\%) & Median OS in months $(95 \% \mathrm{CI})$ & $P$ value* \\
\hline \multicolumn{7}{|l|}{ Age } \\
\hline$<50$ & 5 & 100 & 100 & 75 & $32.7(20.7-44.7)$ & \multirow[t]{2}{*}{0.06} \\
\hline$\geq 50$ & 24 & 63 & 38 & 23 & $16.6(10.1-23.1)$ & \\
\hline \multicolumn{7}{|l|}{ Gender } \\
\hline Female & 11 & 55 & 45 & 27 & $16.7(9.4-24.0)$ & \multirow[t]{2}{*}{0.58} \\
\hline Male & 18 & 78 & 50 & 33 & $18.2(9.8-26.6)$ & \\
\hline \multicolumn{7}{|l|}{ WHO Grade } \\
\hline III $^{\#}$ & 5 & 100 & 100 & 67 & $29.7(13.0-46.4)$ & \multirow[t]{2}{*}{0.04} \\
\hline IV & 24 & 63 & 38 & 26 & $16.6(10.1-23.1)$ & \\
\hline \multicolumn{7}{|c|}{ Extent of resection } \\
\hline Total & 18 & 89 & 56 & 38 & $22.3(12.5-32.1)$ & \multirow[t]{2}{*}{0.37} \\
\hline Subtotal & 11 & 36 & 36 & 20 & $11.4(10.0-12.8)$ & \\
\hline \multicolumn{7}{|c|}{ Karnofsky perfusion status } \\
\hline$\leq 80$ & 18 & 61 & 39 & 28 & $16.4(7.9-24.9)$ & \multirow[t]{2}{*}{0.70} \\
\hline$>80$ & 11 & 82 & 64 & 38 & $24.4(16.9-31.9)$ & \\
\hline \multicolumn{7}{|c|}{ MGMT status (Grade IV patients only) } \\
\hline Methylated & 14 & 93 & 57 & 38 & $18.2(8.7-27.7)$ & \multirow[t]{2}{*}{$<0.001$} \\
\hline Unmethylated & 10 & 20 & 20 & 10 & $10.4(7.5-13.3)$ & \\
\hline \multicolumn{7}{|c|}{ IDH status (Grade III patient only) } \\
\hline Mutated & 4 & 100 & 100 & 100 & 29.7 (not available) & \multirow[t]{2}{*}{0.05} \\
\hline Wild-type & 1 & 100 & 100 & 0 & 18.2 (not available) & \\
\hline \multicolumn{7}{|l|}{ Re-operation } \\
\hline Yes & 6 & 83 & 50 & 0 & $16.7(12.2-21.2)$ & \multirow[t]{2}{*}{0.40} \\
\hline No & 23 & 65 & 52 & 38 & $22.3(12.3-32.3)$ & \\
\hline \multicolumn{7}{|c|}{ Second-line fotemustine chemotherapy (WHO Grade IV patients only) } \\
\hline Yes & 18 & 61 & 33 & 12 & $16.4(8.1-24.7)$ & \multirow[t]{2}{*}{0.24} \\
\hline No & 6 & 67 & 67 & 67 & $28.8(3.8-53.8)$ & \\
\hline
\end{tabular}

OS Overall survival, CI Confidence interval, WHO World Health Organization

* Log-rank $P$ value comparing OS for the demographic factor

\# Anaplastic oligodendroglioma

\section{Serial analysis}

Figure 1a shows the serial changes in the CEL of patients stratified by 18 months OS. Patients appeared to show a reduction in CEL volumes after surgery and radiotherapy compared to baseline, and this was significant for longterm survivors ( $\mathrm{OS} \geq 18$ months) $(P<0.04)$. There were also decreasing trends in BF, BV, and PS in the CEL after surgery and radiotherapy. These trends were significant for $\mathrm{BF}$ and $\mathrm{BV}$ in long-term survivors $(P<0.05$ and $P<0.03$, respectively). In general, CEL volumes, $\mathrm{BF}, \mathrm{BV}$, and $\mathrm{PS}$ appeared to be higher in short-term survivors (OS $<18$ months) compared to long-term survivors. Serial changes in these patients stratified by 12 and 24 months OS are provided in Fig. S1.

Figure $1 \mathrm{~b}$ illustrates the serial changes in the NEL of patients stratified by 18 months OS. NEL volumes decreased after surgery and radiotherapy. The change in NEL volume (compared to baseline) was significant for long-term survivors $(P<0.03)$. However, there was no between-group difference in NEL volumes in long-term versus short-term survivors. BF values in the NEL before surgery were significantly higher in short-term survivors $(P=0.003)$ compared with long-term survivors, and they were also significantly higher at 3 months post-radiotherapy $(P=0.05)$ in short-term survivors compared to long-term survivors. BV values in the NEL were significantly higher at 1 and 3 months post-radiotherapy in short-term survivors $(P=0.04$ and $P=0.02$, respectively) when compared to long-term survivors. PS values in the NEL significantly decreased after surgery and radiotherapy in long-term survivors $(P<0.01$ and $P \leq 0.03$, respectively), and also in short-term survivors $(P<0.04)$. PS values in the NEL at one and three months post- 

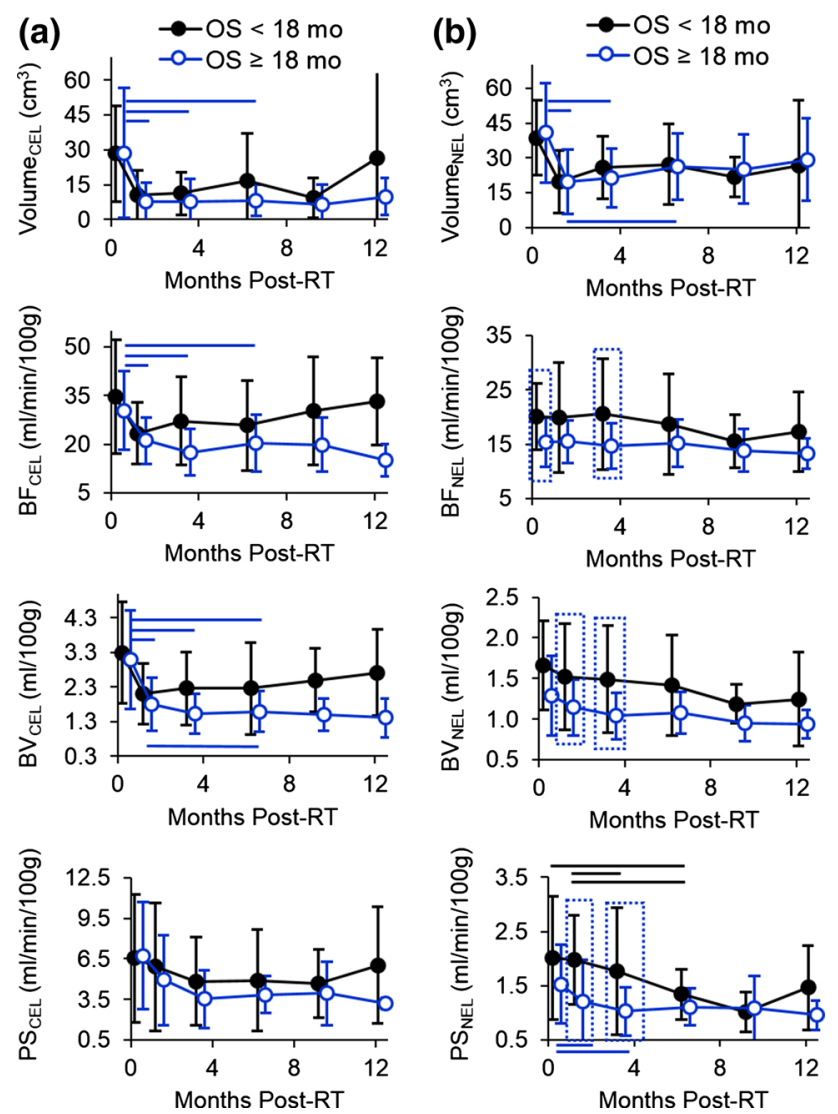

Fig. 1 Serial changes in a the contrast-enhancing lesion (CEL) and b the non-enhancing lesion (NEL) of patients stratified by 18 months (mo) overall survival (OS) for volume (top row), blood flow (BF, second row), blood volume (BV, third row), and permeability-surface area product (PS, last row). Horizontal line connects significant changes between two time points of the same group. Dotted box encloses a significant difference between the groups at a particular time point. Error bar represents $1 \mathrm{SD}$

radiotherapy were significantly higher in short-term survivors when compared to long-term survivors $(P<0.01$ and $P=0.02$, respectively). Figure $\mathrm{S} 2$ shows these serial changes in patients stratified by 12 and 24 months OS.

\section{Survival analysis}

For the entire cohort of patients $(N=29)$, the Cox proportional hazards regression model of the "classical" prognostic factors (i.e. age, performance status, extent of resection, and WHO grade) showed that higher WHO grade $(\mathrm{HR}=49.6,95 \% \mathrm{CI}=5.1-483.2, P<0.001)$ and lower extent of resection $(\mathrm{HR}=19.0,95 \% \mathrm{CI}=3.6-99.5$, $P<0.001)$ were associated with significant hazards of death. Cox proportional hazards regression models of the imaging parameters were evaluated while adjusting for age, performance status, extent of resection and WHO grade. Figure 2 illustrates imaging parameters with cut points that were associated with both significant HRs and significant differences in OS for all 29 patients.

For WHO grade IV glioma patients, Cox proportional hazards regression of MGMT promoter status, age, extent of resection, and performance status showed methylated MGMT promoter status was associated with better OS $(\mathrm{HR}=0.28,95 \% \mathrm{CI}=0.09-9.85, P=0.02)$. With the exception of CEL volume at 6 months post-radiotherapy, the Kaplan-Meier analysis results presented in Fig. 2 were also significant for patients with grade IV gliomas and the Cox proportional hazards regression results were significant even with the adjustment for MGMT status, age, extent of resection, and performance status $(P \leq 0.05)$. In addition, grade IV glioma patients with high BV in the NEL 3 months post-radiotherapy had significantly shorter $\mathrm{OS} \quad(\mathrm{BV} \geq$ $1.1 \mathrm{ml} / 100 \mathrm{~g}, \quad$ median $\mathrm{OS}=11.4,95 \% \mathrm{CI}=$ 9.8-13.0 months) than those with low BV $(\mathrm{BV}<1.1 \mathrm{ml} /$ $100 \mathrm{~g}$, median $\mathrm{OS}=23.6,95 \% \mathrm{CI}=20.3-26.9, \log$-rank $P=0.01$ ), and this was associated with a significant HR 7.4 (95\% CI 1.6-34.6, $P=0.01$ ). Joint modeling of longitudinal covariates and OS showed that changes in any of the imaging parameters did not affect survival.

Table 2 shows the sensitivities and specificities of imaging parameters that are $\geq 70 \%$ in stratifying OS of grade IV glioma patients. BF in the NEL at pre-surgery could stratify patients by 18 and 24 months OS with sensitivities and specificities $\geq 80 \%$. Similarly, BV in the CEL and NEL 3 months post-radiotherapy could stratify patients by 24 months OS with sensitivities and specificities $\geq 80 \%$. It is important to note that volumes of NEL and CEL could not stratify OS with sensitivities and specificities $\geq 70 \%$. The extent of resection could predict 12 months OS with $100 \%$ sensitivity and $78 \%$ specificity, but failed to achieve a specificity of $>50 \%$ when predicting 18 and 24 months OS. MGMT promoter status could predict 12 months OS with $87 \%$ sensitivity and $89 \%$ specificity, but specificities fell below $60 \%$ when predicting 18 and 24 months OS. Figure 3 illustrates representative pre-surgery $\mathrm{CT}$ perfusion and MR images of two patients with different survival (16.7 vs. 41.6 months). Higher BF, BV, and PS in the NEL can be seen in the patient with shorter survival.

\section{Discussion}

In our analysis of patients treated for malignant gliomas, we found that $\mathrm{CT}$ perfusion parameters could potentially identify patients with favorable outcomes based on pretreatment parameters as well as at early time points posttreatment. Based on our findings, we suggest CT perfusion could potentially serve as a valuable imaging biomarker that is complementary to conventional MR imaging. 
(a) Measured at Pre-surgery

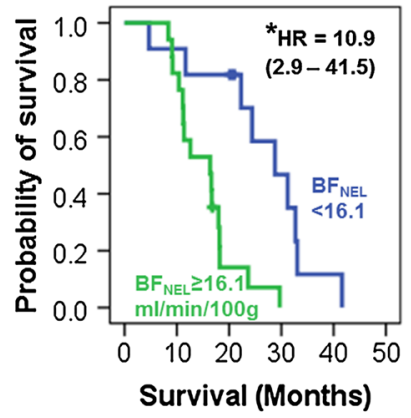

(c) Measured at 3 Months Post-

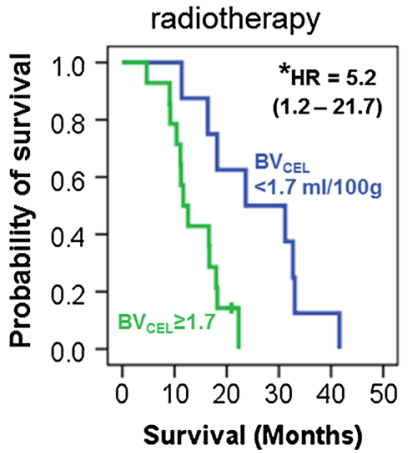

(b)

Measured at 1 Month post-radiotherapy

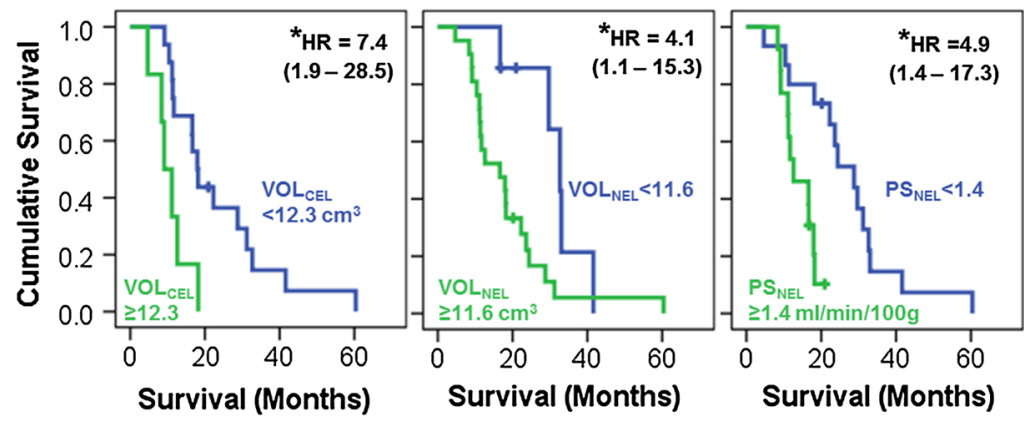

(d) Measured at 6 Months Post-radiotherapy

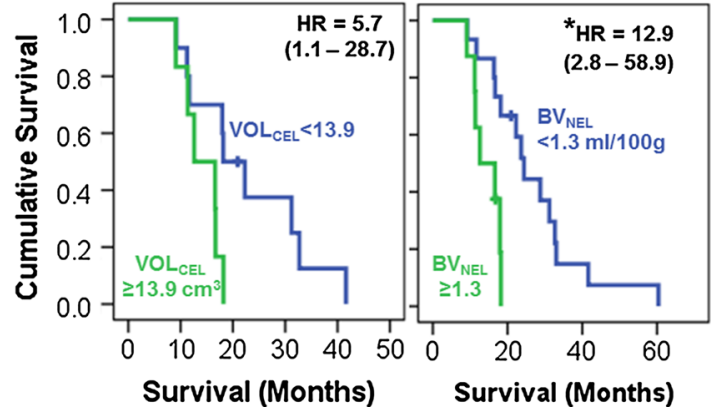

Fig. 2 Kaplan-Meier survival plots of a blood flow in the nonenhancing lesion $\left(\mathrm{BF}_{\mathrm{NEL}}\right)$ measured at pre-surgery, $\mathbf{b}$ volume of the contrast-enhancing lesion $\left(\mathrm{VOL}_{\mathrm{CEL}}\right)$, volume in the NEL $\left(\mathrm{VOL}_{\mathrm{NEL}}\right)$, permeability-surface area product in the NEL $\left(\mathrm{PS}_{\mathrm{NEL}}\right)$ measured at 1 month post-radiotherapy, c blood volume in the CEL $\left(\mathrm{BV}_{\mathrm{CEL}}\right)$ measured at 3 months post-radiotherapy, and $\mathbf{d} \mathrm{VOL}_{\mathrm{CEL}}$ and $\mathrm{BV}$ in the NEL $\left(\mathrm{BV}_{\mathrm{NEL}}\right)$ measured at 6 months post-radiotherapy for all patients $(N=29)$. Higher values in these parameters were associated with worse overall survival (log-rank $P<0.05$ for all comparisons).

We first investigated the serial changes in volume, BF, $\mathrm{BV}$, and PS. The initial decline of all imaging parameters in the CEL after surgery and radiotherapy could be attributed to surgical debulking of the tumor and therapeutic effect of radiotherapy and chemotherapy. After stratifying patients based on OS, all three perfusion parameters in the CEL started to diverge as early as 3 months after radiotherapy with elevated $\mathrm{BF}, \mathrm{BV}$, and PS seen in patients with shorter OS. BF, BV, and PS in the NEL were also higher in patients with shorter OS. Although statistical differences were identified only at selected time points, our data provided corroborating evidence that the variation in tumor perfusion characteristics in the CEL and NEL were associated with differences in OS. This variation in perfusion characteristics reflects differences in angiogenesis and vascularity (and by extension differences in aggressiveness and treatment response) between patients. Angiogenesis leads to an increase in tumor microvessel area that facilitates tumor growth, and it is an active target in cancer therapies [28]. It has been shown to correlate with tumor
These parameters were associated with significant hazard ratios (HR) after adjusting for tumor grade, age, Karnofsky performance status, and extent of surgical resection $(P \leq 0.05)$. Numbers in parentheses are the $95 \%$ confidence interval (CI) of the HR. Asterisk represents significant HR and significantly different OS when considering only grade IV glioma patients with adjustments for MGMT promoter status, age, extent of resection, and Karnofsky performance status in the Cox proportional hazard regression

BV and patient survival [10]. More recently, vasculogenesis, a process by which bone marrow-derived cells are recruited to form new tumor blood vessels, has been shown to govern tumor recurrence after radiotherapy [29]. Given that recurrence occurs within $2 \mathrm{~cm}$ of the irradiated volume [30], it is critical to assess perfusion parameters in both the CEL and NEL. In fact, we found perfusion parameters in the CEL and NEL to be prognostic of OS. The importance of perfusion parameters in the NEL reflect the limitation of conventional contrast-enhanced MR where enhancement may be more reflective of changes in vessel architecture and permeability (i.e. breakdown of the blood-brain barrier) rather than reflecting the overall vascular supply to the tumor.

None of the imaging measurements made in the CEL before surgery showed a significant association with OS. This could be because total resection of the tumor (mostly CEL) is a stronger predictor of OS. Over $60 \%$ of patients underwent total resection, and it showed a significant association with OS. Prediction of survival using pre-surgery 
Table 2 Receiver operating characteristic analysis of imaging parameters with sensitivities and specificities $\geq 70 \%$ in stratifying overall survival (OS) for patients with grade IV gliomas only

\begin{tabular}{|c|c|c|c|c|c|c|}
\hline Imaging Time & Cut-point selected based on & Region & Parameter cut point & AUC & Sensitivity (\%) & Specificity $(\%)$ \\
\hline \multirow[t]{2}{*}{ Pre-surgery } & $18 \mathrm{mo} \mathrm{OS}^{\mathrm{a}}$ & NEL & $\mathrm{BF} \geq 16.8 \mathrm{ml} / \mathrm{min} / 100 \mathrm{~g}$ & 0.93 & 80 & 100 \\
\hline & $24 \mathrm{mo} \mathrm{OS}^{\mathrm{b}}$ & NEL & $\mathrm{BF} \geq 16.1 \mathrm{ml} / \mathrm{min} / 100 \mathrm{~g}$ & 0.95 & 82 & 100 \\
\hline \multirow[t]{3}{*}{1 month post-radiotherapy } & $18 \mathrm{mo} \mathrm{OS}^{\mathrm{a}}$ & NEL & $\mathrm{PS} \geq 1.3 \mathrm{ml} / \mathrm{min} / 100 \mathrm{~g}$ & 0.86 & 79 & 89 \\
\hline & $24 \mathrm{mo} \mathrm{OS}^{\mathrm{a}}$ & CEL & $\mathrm{PS} \geq 2.3 \mathrm{ml} / \mathrm{min} / 100 \mathrm{~g}$ & 0.82 & 93 & 75 \\
\hline & $24 \mathrm{mo} \mathrm{OS}$ & NEL & $\mathrm{BV} \geq 1.1 \mathrm{ml} / 100 \mathrm{~g}$ & 0.80 & 75 & 83 \\
\hline \multirow[t]{8}{*}{3 months post-radiotherapy } & $12 \mathrm{mo} \mathrm{OS}$ & NEL & $\mathrm{BF} \geq 14.8 \mathrm{ml} / \mathrm{min} / 100 \mathrm{~g}$ & 0.67 & 78 & 71 \\
\hline & $12 \mathrm{mo} \mathrm{OS}$ & NEL & $\mathrm{BV} \geq 1.1 \mathrm{ml} / 100 \mathrm{~g}$ & 0.73 & 78 & 71 \\
\hline & $18 \mathrm{mo} \mathrm{OS}$ & CEL & $\mathrm{BV} \geq 1.7 \mathrm{ml} / 100 \mathrm{~g}$ & 0.76 & 86 & 71 \\
\hline & $18 \mathrm{mo} \mathrm{OS}$ & NEL & $\mathrm{BF} \geq 14.8 \mathrm{ml} / \mathrm{min} / 100 \mathrm{~g}$ & 0.79 & 71 & 89 \\
\hline & $18 \mathrm{mo} \mathrm{OS}$ & NEL & $\mathrm{BV} \geq 1.1 \mathrm{ml} / 100 \mathrm{~g}$ & 0.81 & 71 & 89 \\
\hline & $24 \mathrm{mo} \mathrm{OS}^{\mathrm{b}}$ & CEL & $\mathrm{BV} \geq 1.7 \mathrm{ml} / 100 \mathrm{~g}$ & 0.84 & 81 & 100 \\
\hline & $24 \mathrm{mo} \mathrm{OS}$ & NEL & $\mathrm{BF} \geq 14.4 \mathrm{ml} / \mathrm{min} / 100 \mathrm{~g}$ & 0.73 & 75 & 83 \\
\hline & $24 \mathrm{mo} \mathrm{OS}$ & NEL & $\mathrm{BV} \geq 1.0 \mathrm{ml} / 100 \mathrm{~g}$ & 0.84 & 81 & 83 \\
\hline 6 months post-radiotherapy & $12 \mathrm{mo} \mathrm{OS}$ & NEL & $\mathrm{BV} \geq 1.2 \mathrm{ml} / 100 \mathrm{~g}$ & 0.76 & 83 & 71 \\
\hline
\end{tabular}

$N E L$ non-enhancing lesion, $C E L$ contrast-enhancing lesion; $m o$ months, $O S$ overall survival, $B F$ blood flow, $B V$ blood volume, $P S$ permeabilitysurface area product, $A U C$ area under the receiver operating characteristic curve

a Parameters with sensitivities and specificities $\geq 70 \%$ in stratifying OS when considering both grade III and IV glioma patients

b Parameters with sensitivities and specificities $\geq 80 \%$ in stratifying OS when considering both grade III and IV glioma patients

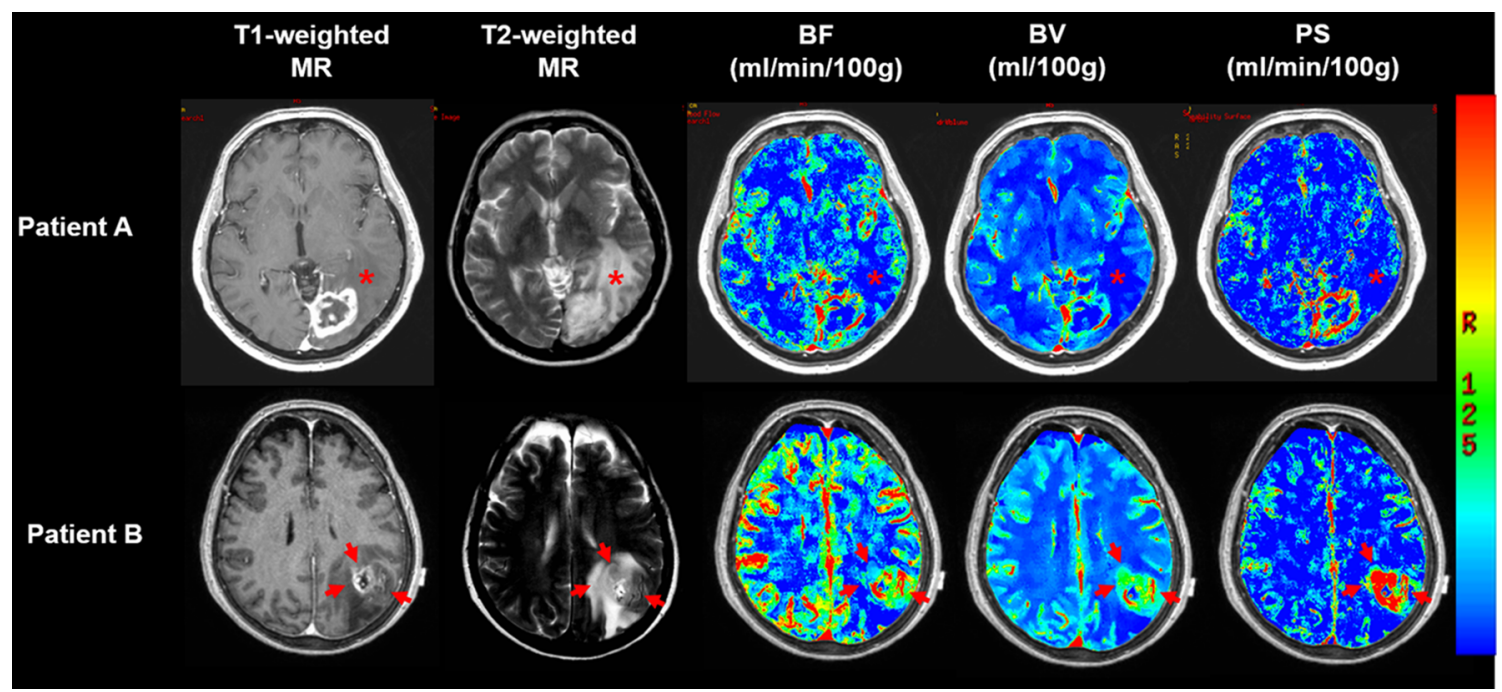

Fig. 3 Illustrative pre-surgery CT perfusion and MR images of patients with WHO grade IV gliomas. Both patients presented with a contrast-enhancing lesion on post-gadolinium T1-weighted MR images, which also had elevated blood flow (BF), blood volume (BV), and permeability-surface area product (PS). Patient A presented

CT perfusion parameters in the CEL may be more appropriate for patients who are not candidates for total resection. For example, pre-surgery tumor BV is a predictor of survival in a study with a mixture of patients that received biopsy, subtotal resection, and total resection [11]. However, pre-surgery measurement of BF in the NEL proved to be useful in predicting OS in this study. This result points with low BF, BV, and PS in the non-enhancing lesion (NEL, red asterisks). However, Patient $\mathrm{B}$ presented with regions of elevated $\mathrm{BF}, \mathrm{BV}$, and PS in the NEL (red arrows). The survival for patient A was 41.6 months, and patient B was 16.7 months. Identical window and level were used for the color maps

to the significance of tumor burden in the $\mathrm{T} 2$ hyperintense lesion that is not typically removed by surgery.

Although a larger sample size is required to show consistent associations between OS and CT perfusion parameters across all time points, our results are consistent with previous reports that higher BV and PS are associated with poor outcomes $[11,12,20,31,32]$. It is also 
noteworthy that while we showed significant hazards of death associated with both volumetric and CT perfusion parameters, only CT perfusion parameters resulted in sensitivities and specificities $\geq 70 \%$ in stratifying OS while volumetric parameters did not. Volume of the CEL may not be a reliable predictor of outcome because the volume of contrast-enhancement may be affected by many nontumoral processes including inflammation, postsurgical changes, pseudoprogression, and treatment-induced necrosis [33-36]. Similarly, the volume of the NEL encompasses many causes of $\mathrm{T} 2$ hyperintensity such as vasogenic edema, gliosis, cystic changes, inflammation, and tumor infiltration [37-39]. It is evident from Fig. 3 that tumors can have regions of high $\mathrm{BF}, \mathrm{BV}$, and $\mathrm{PS}$ that are bigger than the CEL. These regions can potentially lead to future sites of recurrence. Although this is hypothesisgenerating, there is preliminary evidence to suggest that there could be better spatial concordance between CT perfusion parameters with the site of future recurrence than the concordance between CEL and the site of future recurrence [24].

Some limitations must be noted. Firstly, a one-phase rather than two-phase $\mathrm{CT}$ perfusion protocol was used in one-third of the patients. Although a shorter scan duration of the CT perfusion protocol can affect measurements of PS, the effects on BF and BV are smaller [25]. The identified predictors of OS are unlikely to change, but the cut points of these predictors could change with a larger cohort of patients studied with a uniform two-phase protocol. It is important to use a two-phase CT perfusion protocol in future studies. Secondly, we could not adjust for the effect of IDH1 mutation status in our regression models due to a small number of WHO grade III glioma patients. Finally, a CT perfusion scan at the time point of pre-radiotherapy but post-surgery could be a better baseline scan than a presurgery perfusion imaging scan. The prognostic importance of CT perfusion parameters obtained pre-radiotherapy compared to post-radiotherapy remains to be demonstrated.

\section{Conclusions}

$\mathrm{BF}, \mathrm{BV}$, and PS are potential biomarkers of OS in patients with high-grade gliomas treated with multi-modality therapies (surgery, radiotherapy, chemotherapy) even after adjustments for age, WHO grade, Karnofsky performance status, the extent of resection, and MGMT promoter status. The results of this study, if verified in a larger cohort of patients, could establish CT perfusion imaging as a reliable early predictor of survival.

Acknowledgments This project was funded by The Project of Emilia-Romagna region on Neuro-Oncology (PERNO) study group, the Canadian Institutes of Health Research (CIHR), and the CIHR Strategic Training Program in Cancer Research and Technology Transfer.

Funding Ting-Yim Lee licenses CT Perfusion software to and receives funding from GE Healthcare.

\section{Appendix}

\section{Steering committee}

Baruzzi A. (Chair), Albani F., Calbucci F., D’Alessandro R., Michelucci R. (IRCCS Institute of Neurological Sciences, Bologna, Italy), Brandes A. (Department of Medical Oncology, Bellaria-Maggiore Hospitals, Bologna, Italy), Eusebi V. (Department of Hematology and Oncological Sciences “L. \& A. Seràgnoli", Section of Anatomic Pathology at Bellaria Hospital, Bologna, Italy), Ceruti S., Fainardi E., Tamarozzi R. (Neuroradiology Unit, Department of Neurosciences and Rehabilitation, S. Anna Hospital, Ferrara, Italy), Emiliani E. (Istituto Oncologico Romagnolo, Department of Medical Oncology, Santa Maria delle Croci Hospital, Ravenna, Italy), Cavallo M. (Division of Neurosurgery, Department of Neurosciences and Rehabilitation, S. Anna Hospital, Ferrara, Italy).

\section{Executive committee}

Franceschi E., Tosoni A. (Department of Medical Oncology, Bellaria-Maggiore Hospitals, Bologna, Italy), Cavallo M. (Division of Neurosurgery, Department of Neurosciences and Rehabilitation, S. Anna Hospital, Ferrara, Italy), Fiorica F. (Department of Radiation Oncology, S. Anna Hospital, Ferrara, Italy), Valentini A. (Division of Neurosurgery, Nuovo Ospedale Civile S. AgostinoEstense, Baggiovara, Modena, Italy), Depenni R. (Department of Oncology, Policlinico di Modena, Italy), Mucciarini C. (Department of Oncology, Ramazzini Hospital, Carpi, Modena, Italy), Crisi G. (Department of Neuroradiology, Maggiore Hospital, Parma, Italy), Sasso E. (Department of Neurological Sciences, Maggiore Hospital, Parma, Italy), Biasini C., Cavanna L. (Department of Oncology and Hematology, Guglielmo da Saliceto Hospital, Piacenza, Italy), Guidetti D. (Department of Neurology, Guglielmo da Saliceto Hospital, Piacenza, Italy), Marcello N., Pisanello A. (Department of Neurology, Istituto in tecnologie avanzate e modelli assistenziali in oncologia, IRCCS, S. Maria Nuova Hospital, Reggio Emilia, Italy), Cremonini A.M., Guiducci G. (Division of Neurosurgery, M. Bufalini Hospital, Cesena, Italy).

Registry Coordination Office: de Pasqua S., Testoni S. (IRCCS Institute of Neurological Sciences, Bologna, Italy). 


\section{Participants}

Agati R., Ambrosetto G., Bacci A., Baldin E., Baldrati A., Barbieri E., Bartolini S., Bellavista E., Bisulli F., Bonora E., Bunkheila F., Carelli V., Crisci M., Dall'Occa P., Ferro S., Franceschi C., Frezza G., Grasso V., Leonardi M., Morandi L., Mostacci B., Palandri G., Pasini E., Pastore Trossello M., Poggi R., Riguzzi P., Rinaldi R., Rizzi S., Romeo G., Spagnolli F., Tinuper P., Trocino C. (Bologna), Dall'Agata M., Frattarelli M., Gentili G., Giovannini A., Iorio P., Pasquini U., Galletti G., Guidi C., Neri W., Patuelli A., Strumia S. (Forlì-Cesena), Faedi M. (IRCCS Istituto Scientifico Romagnolo per lo Studio e la Cura dei Tumori), Casmiro M., Gamboni A., Rasi F. (Faenza R.A.), Cruciani G. (Lugo, RA), Cenni P., Dazzi C., Guidi A.R., Zumaglini F. (Ravenna), Amadori A., Pasini G., Pasquinelli M., Pasquini E., Polselli A., Ravasio A., Viti B. (Rimini), Sintini M. (Cattolica, RN), Ariatti A., Bertolini F., Bigliardi G., Carpeggiani P., Cavalleri F., Meletti S., Nichelli P., Pettorelli E., Pinna G., Zunarelli E. (Modena), Artioli F., Bernardini I., Costa M., Greco G., Guerzoni R., Stucchi C. (Carpi M.O.), Iaccarino C., Ragazzi M., Rizzi R., Zuccoli G. (Istituto di Ricovero e Cura a Carattere Scientifico, Reggio Emilia), Api P., Cartei F., Colella M., Fallica E., Farneti M., Frassoldati A., Granieri E., Latini F., Monetti C., Saletti A., Schivalocchi R., Sarubbo S., Seraceni S., Tola M.R., Urbini B., Zini G. (Ferrara), Giorgi C., Montanari E. (Fidenza P.R.), Cerasti D., Crafa P., Dascola I., Florindo I., Giombelli E., Mazza S., Ramponi V., Servadei F., Silini E.M., Torelli P. (Parma), Immovilli P., Morelli N., Vanzo C. (Piacenza), Nobile C. (Padova).

\section{References}

1. Wen PY, Kesari S (2008) Malignant gliomas in adults. N Engl J Med 359:492-507

2. Curran WJ, Scott CB, Horton J et al (1993) Recursive partitioning analysis of prognostic factors in three radiation therapy oncology group malignant glioma trials. J Natl Cancer Inst 85:704-710

3. Weller M, Stupp R, Hegi ME et al (2012) Personalized care in neuro-oncology coming of age: why we need MGMT and 1p/19q testing for malignant glioma patients in clinical practice. Neuro Oncol 14(suppl iv):100-108

4. Wen PY, Macdonald DR, Reardon DA et al (2010) Updated response assessment criteria for high-grade gliomas: response assessment in neuro-oncology working group. J Clin Oncol 28:1963-1972

5. Essig M, Anzalone N, Combs SE et al (2012) MR imaging of neoplastic central nervous system lesions: Review and recommendations for current practice. Am J Neuroradiol 33:803-817

6. Cha S (2006) Update on brain tumor imaging: from anatomy to physiology. Am J Neuroradiol 27:475-487

7. Jain R, Ellika SK, Scarpace L et al (2008) Quantitative estimation of permeability surface-area product in astroglial brain tumors using perfusion CT and correlation with histopathologic grade. Am J Neuroradiol 29:694-700
8. Weber MA, Henze M, Tüttenberg J et al (2010) Biopsy targeting gliomas: do functional imaging techniques identify similar target areas? Invest Radiol 45:755-768

9. Jain R, Gutierrez J, Narang J et al (2011) In vivo correlation of tumor blood volume and permeability with histologic and molecular angiogenic markers in gliomas. Am J Neuroradiol 32:388-394

10. Hu LS, Eschbacher JM, Dueck AC et al (2012) Correlations between perfusion MR imaging cerebral blood volume, microvessel quantification, and clinical outcome using stereotactic analysis in recurrent high-grade glioma. Am J Neuroradiol 33:69-76

11. Jain R, Narang J, Griffith B et al (2013) Prognostic vascular imaging biomarkers in high-grade gliomas: tumor permeability as an adjunct to blood volume estimates. Acad Radiol 20:478-485

12. Shankar JJ, Woulfe J, Silva VD, Nguyen TB (2013) Evaluation of perfusion $\mathrm{CT}$ in grading and prognostication of high-grade gliomas at diagnosis: a pilot study. AJR Am J Roentgenol 200:W504-W509

13. Law M, Young RJ, Babb JS et al (2008) Gliomas: predicting time to progression or survival with cerebral blood volume measurements at dynamic susceptibility-weighted contrast-enhanced perfusion MR imaging. Radiology 247:490-498

14. Bisdas S, Kirkpatrick M, Giglio P, Welsh C, Spampinato MV, Rumboldt Z (2009) Cerebral blood volume measurements by perfusion-weighted MR imaging in gliomas: ready for prime time in predicting short-term outcome and recurrent disease? Am J Neuroradiol 30:681-688

15. Galbán CJ, Chenevert TL, Meyer CR et al (2011) Prospective analysis of parametric response map-derived MRI biomarkers: Identification of early and distinct glioma response patterns not predicted by standard radiographic assessment. Clin Cancer Res $17: 4751-4760$

16. Jain R (2011) Perfusion CT imaging of brain tumors: an overview. AJNR Am J Neuroradiol 32:1570-1577

17. Ding B, Ling HW, Chen KM, Jiang H, Zhu YB (2006) Comparison of cerebral blood volume and permeability in preoperative grading of intracranial glioma using CT perfusion imaging. Neuroradiology 48:773-781

18. Fainardi E, Di Biase F, Borrelli M et al (2010) Potential role of CT perfusion parameters in the identification of solitary intraaxial brain tumor grading. Acta Neurochir Suppl 106:283-287

19. Ellika SK, Jain R, Patel SC et al (2007) Role of perfusion CT in glioma grading and comparison with conventional MR imaging features. Am J Neuroradiol 28:1981-1987

20. Jain R, Narang J, Schultz L et al (2011) Permeability estimates in histopathology-proved treatment-induced necrosis using perfusion CT: Can these add to other perfusion parameters in differentiating from recurrent/progressive tumors? Am J Neuroradiol 32:658-663

21. Vidiri A, Guerrisi A, Pinzi V et al (2012) Perfusion Computed Tomography (PCT) adopting different perfusion metrics: recurrence of brain metastasis or radiation necrosis? Eur J Radiol $81: 1246-1252$

22. Herman JG, Graff JR, Myöhänen S, Nelkin BD, Baylin SB (1996) Methylation-specific PCR: a novel PCR assay for methylation status of $\mathrm{CpG}$ islands. Proc Natl Acad Sci USA 93:9821-9826

23. Hartmann C, Meyer J, Balss J et al (2009) Type and frequency of IDH1 and IDH2 mutations are related to astrocytic and oligodendroglial differentiation and age: a study of 1,010 diffuse gliomas. Acta Neuropathol 118:469-474

24. Yeung TPC, Yartsev Y, Lee TY et al (2014) Relationship of computed tomography perfusion and positron emission tomography to tumour progression in malignant glioma. J Med Radiat Sci 61:4-13 
25. Yeung TPC, Yartsev S, Bauman G, He W, Fainardi E, Lee TY (2013) The effect of scan duration on the measurement of perfusion parameters in CT perfusion studies of brain tumors. Acad Radiol 20:59-65

26. Pieper S, Lorensen B, Schroeder W, Kikinis R (2006) The NAMIC Kit: ITK, VTK, pipelines, grids and 3D slicer as an open platform for the medical image computing community. In: Proceedings of the 3rd IEEE international symposium biomedical imaging: From Nano Macro, vol 1, pp 698-701

27. Rizopoulos D (2010) JM: an R package for the joint modelling of longitudinal and time-to-event data. J Stat Softw 35:1-33

28. Jain RK, di Tomaso E, Duda DG, Loeffler JS, Sorensen AG, Batchelor TT (2007) Angiogenesis in brain tumours. Nat Rev Neurosci 8:610-622

29. Kioi M, Vogel H, Schultz G, Hoffman RM, Harsh GR, Brown JM (2010) Inhibition of vasculogenesis, but not angiogenesis, prevents the recurrence of glioblastoma after irradiation in mice. J Clin Invest 120:694-705

30. Milano MT, Okunieff P, Donatello RS et al (2010) Patterns and timing of recurrence after temozolomide-based chemoradiation for glioblastoma. Int J Radiat Oncol Biol Phys 78:1147-1155

31. Vöglein J, Tüttenberg J, Weimer M et al (2011) Treatment monitoring in gliomas: comparison of dynamic susceptibilityweighted contrast-enhanced and spectroscopic MRI techniques for identifying treatment failure. Invest Radiol 46:390-400

32. Mangla R, Singh G, Ziegelitz D et al (2010) Changes in relative cerebral blood volume 1 month after radiation-temozolomide therapy can help predict overall survival in patients with glioblastoma. Radiology 256:575-584

33. van den Bent MJ, Vogelbaum MA, Wen PY, Macdonald DR, Chang SM (2009) End point assessment in gliomas: novel treatments limit usefulness of classical Macdonald's Criteria. J Clin Oncol 27:2905-2908

34. Finn MA, Blumenthal DT, Salzman KL et al (2007) Transient postictal MRI changes in patients with brain tumors may mimic disease progression. Surg Neurol 67:246-250

35. Ulmer S, Braga TA, Barker FG 2nd et al (2006) Clinical and radiographic features of peritumoral infarction following resection of glioblastoma. Neurology 67:1668-1670

36. Kumar AJ, Leeds NE, Fuller GN et al (2000) Malignant gliomas: MR imaging spectrum of radiation therapy- and chemotherapyinduced necrosis of the brain after treatment. Radiology 217:377-384

37. Oh J, Cha S, Aiken AH et al (2005) Quantitative apparent diffusion coefficients and T2 relaxation times in characterizing contrast enhancing brain tumors and regions of peritumoral edema. J Magn Reson Imaging 21:701-708

38. Hattingen E, Jurcoane A, Daneshvar K et al (2013) Quantitative T2 mapping of recurrent glioblastoma under Bevacizumab improves monitoring for non-enhancing tumor progression and predicts overall survival. Neuro Oncol 15:1395-1404

39. Li Y, Lupo JM, Polley MY et al (2011) Serial analysis of imaging parameters in patients with newly diagnosed glioblastoma multiforme. Neuro Oncol 13:546-557 